\title{
Busy doing nothing: Evidence for nonaction-effect binding
}

\author{
SIMONE KÜHN \\ Max Planck Institute for Human Cognitive and Brain Sciences, Leipzig, Germany \\ and Ghent University, Ghent, Belgium \\ BIRGIT ELSNER \\ University of Potsdam, Golm, Germany \\ Wolfgang Prinz \\ Max Planck Institute for Human Cognitive and Brain Sciences, Leipzig, Germany \\ AND \\ Marcel Brass \\ Ghent University, Ghent, Belgium
}

\begin{abstract}
Research on voluntary action has focused on the question of how we represent our behavior on a motor and cognitive level. However, the question of how we represent voluntary not acting has been completely neglected. The aim of the present study was to investigate the cognitive and motor representation of intentionally not acting. By using an action-effect binding approach, we demonstrate similarities of action and nonaction. In particular, our results reveal that voluntary nonactions can be bound to an effect tone. This finding suggests that effect binding is not restricted to an association between a motor representation and a successive effect (action-effect binding) but can also occur for an intended nonaction and its effect (nonaction-effect binding). Moreover, we demonstrate that nonactions have to be initiated voluntarily in order to elicit nonaction-effect binding.
\end{abstract}

Daily life involves various incidents of intentional nonaction. Imagine a wife demanding to talk with her spouse about their relationship and him ignoring her request. Presumably this not-acting is a voluntary act. And very often intentional nonactions ${ }^{1}$ have foreseeable consequences. If, for instance, you decide not to catch a ball flying straight at your face, you can anticipate what will happen. Along the same lines, the Taoist concept of wei wu wei, meaning "acting by not acting" (Loy, 1985), considers nonaction to be dynamic and clearly separate from inaction. In the legal domain, human societies acknowledge nonaction (namely negligence) as an intentional act by considering it to be punishable under the precondition of purposefulness.

Surprisingly, psychological research on human performance focuses entirely on the investigation of action, while neglecting intentional nonaction almost completely. This is presumably due to the fact that in experiments on intentional nonaction, one loses the typical dependent measures of experimental psychology - namely, reaction times (RTs) and error rates. In order to fill this gap, we aim to investigate the hypothesis that intentional nonaction shares properties with intentional action, by testing whether both are coded in a similar way.
An influential theory of motor control, ideomotor theory, states that actions are represented in the form of sensory feedback they produce (Greenwald, 1970; Prinz, 1997). In other words, we control our actions by anticipating the sensory consequences of these actions. In accordance with the ideomotor principle, Elsner and Hommel (2001) demonstrated that participants indeed form action-effect associations. In their experiments, participants freely chose between two actions (pressing a right or left key) that were followed by specific but irrelevant effect tones (high- and low-pitched tones). In a test phase, participants were required to respond to the effect tones by choosing spontaneously which button to press. In accordance with ideomotor theory, participants preferred to choose actions that had previously been associated with the tone (consistent mapping), rather than actions that were associated with the other tone (inconsistent responses).

In the present study, we use action-effect binding to investigate whether intentional nonaction shares essential coding properties with intentional action. At the same time, we want to test whether action-effect binding consists of an association between motor patterns and the sensory effect or higher level representations and the subsequent effect.

S. Kühn, skuehn@cbs.mpg.de 
For that purpose, we use the same setup as did Elsner and Hommel (2001, Experiment 2A), with the response option to press the right or left response button and the additional option to press no button. On the basis of the assumption that intentional nonactions resemble intentional actions in that they both involve a voluntary intention, we expect to find action-effect binding - namely, more consistent as opposed to inconsistent choices in a free-choice test phase-for voluntary nonactions as well. This would clearly argue against the assumption that action-effect binding consists of a binding between motor patterns and the effect tones. In Experiment 2, we explore whether the defining feature resulting in the observed binding effect is the deliberateness of the nonaction. We compare a condition in which participants intentionally select not to respond with a condition in which participants are instructed not to respond. As a result, we present evidence indicating that action-effect binding is established if an intentional nonaction leads to effects in the environment but not if an instructed nonaction does.

\section{EXPERIMENT 1}

\section{Method}

Participants. Twelve individuals ( 8 females and 4 males; ages 20-25 years) took part in this experiment. Each participant was paid $€ 7$ for $45 \mathrm{~min}$ of participation. All participants were right-handed and had normal or corrected-to-normal vision.

Experimental Design, Materials, and Procedure. For the acquisition phase, participants were instructed to decide between pressing the right button (right index finger), the left button (left index finger), and no button whenever a white square appeared in the middle of the screen (Figure 1).

The participants were asked to aim at an equal distribution of all three response options without having a certain rule of responding. We asked them to imagine the situation as throwing a die with three sides. We used an intertrial interval of $1,000,1,500$, or $2,000 \mathrm{msec}$, and then presented a white fixation cross on a black background for $100 \mathrm{msec}$, which was followed by a blank screen for $100 \mathrm{msec}$. Then a white square appeared in the middle of the screen for $200 \mathrm{msec}$, which signaled that a decision was to be made. When the participants responded, we presented a tone $250 \mathrm{msec}$ after the response. With reference to the history of RTs in response trials, we determined the interval before the tone presentation for no-buttonpress trials. This interval allows a certain variance in response RTs but stillas participants reported in the debriefing - suggests the feeling of causation of the tone in the nonaction case. The tones we used were $400-, 600-$, and $800-\mathrm{Hz}$ sinusodial wave tones of 200-msec duration delivered via Sennheiser headphones. The action-effect mapping was balanced across participants.

In the test phase, participants were instructed to respond spontaneously to one of the three tones of the acquisition phase with either a right, left, or no buttonpress. We again used an intertrial interval of $1,000,1,500$, or $2,000 \mathrm{msec}$ and then presented a white fixation cross on a black background for $100 \mathrm{msec}$, which was followed by a blank screen for $100 \mathrm{msec}$, after which one of the three tones of 200-msec duration was presented. If the response occurred during the tone presentation, the trials were excluded from further analysis. Likewise, RTs above 1,500 msec were discarded.

Each block consisted of 45 trials; the experiment contained 15 blocks. The block sequence was AAAAAAATTATTATT, where $A$ denotes acquisition blocks and $T$ denotes test blocks. Before the experimental blocks, the participants were briefly familiarized with both tasks by encountering 8 trials of each type.

The data analysis focuses mainly on the test phase. Because of the redundancy of consistent and inconsistent choices, we concentrated the analysis on the frequency (in percent) of consistent choices. We conducted Kolmogorov-Smirnov (KS) tests to exclude violations of the assumption that the frequencies of consistent choices are normally distributed. Then we used $t$ tests to find out whether the frequency of consistent response choices for each tone deviates from chance (.50) (Elsner \& Hommel, 2001).

\section{Results and Discussion}

In the acquisition phase, participants produced, on average, the same number of right, left, and no buttonpresses (right, 135; left, 134; intentional nonaction, 136), confirming that the participants experienced the actioneffect couplings about equally often.

We excluded $4.8 \%$ of the test phase trials because the participants either responded too early $(3.6 \%)$ or too late $(1.2 \%)$. KS tests revealed no violations of the assump-

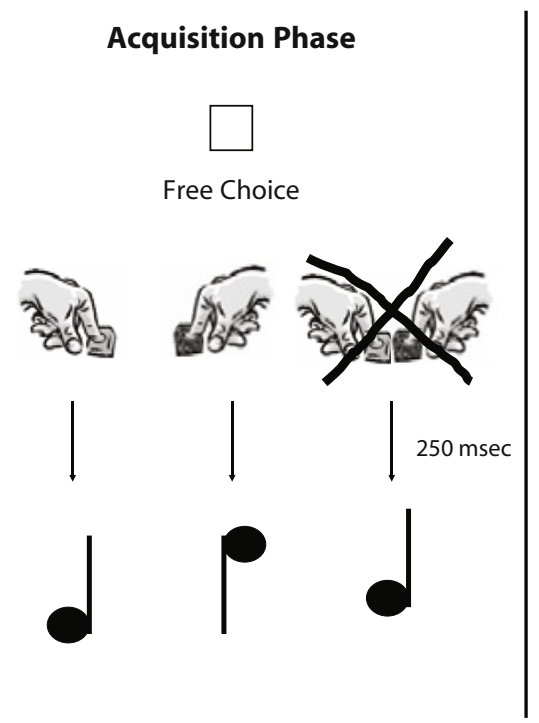

Test Phase

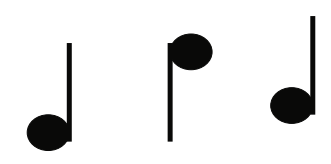

Free Choice

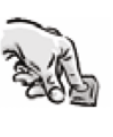

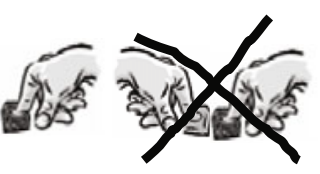

Figure 1. Schematic drawing of the conditions in Experiment 1. 


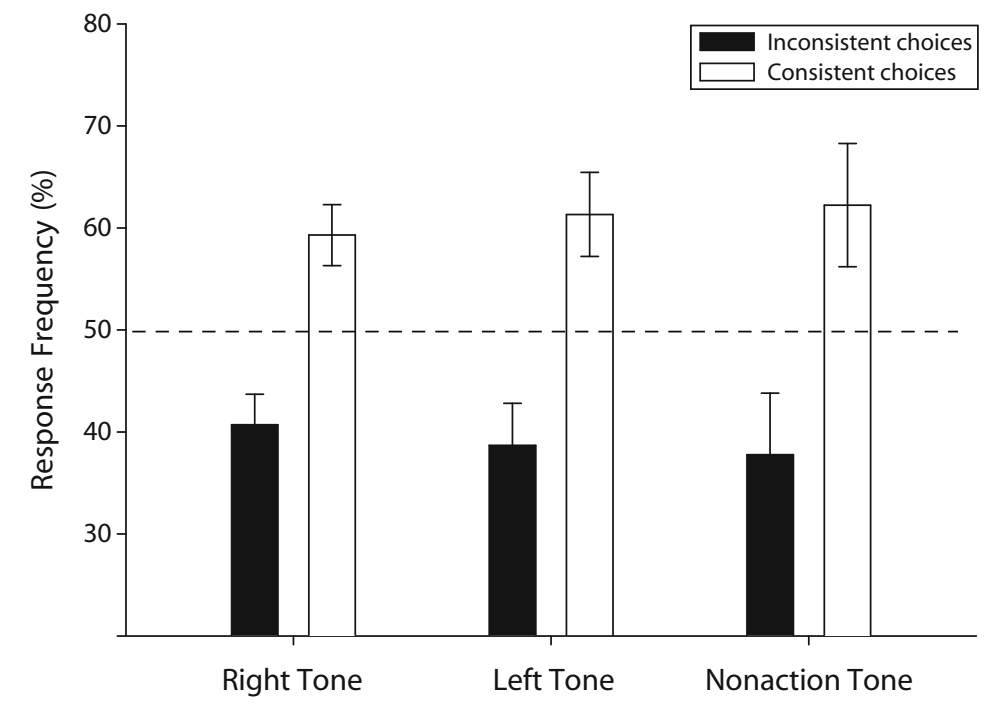

Figure 2. Response frequency in response to the presented tone in Experiment 1. Error bars represent the standard errors of the means.

tion of normal distribution $(p>.47$ for right, left, and nonaction frequency of consistent choices). Consistent with previous work, more detailed $t$ tests revealed that the frequency of consistent choices deviated significantly from chance for the right tone $[t(11)=2.85, p<.05]$ and the left tone $[t(11)=2.35, p<.05]$. Most importantly, a significant consistency effect was also found for the intentional nonaction tone $[t(11)=3.98, p<.01]$ (see Figure 2).

This indicates that participants formed a nonactioneffect association. In summary, the results imply that the perception of an acquired action effect primes the respective action or nonaction representation. This suggests that intentional nonaction shares characteristic properties with intentional action in the context of action-effect binding.

One potential criticism of the first experiment might be that the dependent variable-namely, frequency of choice - could be sensitive to explicit reflections of participants regarding the relationship of action and effect. In the second experiment, we set out to replicate this nonactioneffect binding by means of RTs in a priming task.

\section{EXPERIMENT 2}

In this experiment, participants had to perform a simple two-choice response task in the test phase. When the indicative stimuli appeared, we additionally presented irrelevant compatible and incompatible effect tones (Ziessler $\&$ Nattkemper, 2002). We expected that the former effect tone (a compatible tone) should facilitate the response, whereas the nonaction as well as the action tone associated with the other response should interfere with the afforded response.

\section{Method}

Participants. Fourteen individuals ( 9 females and 5 males; ages 19-27 years) participated in this experiment. Each participant was paid $€ 7$ for 45 min of participation. All participants were righthanded and had normal or corrected-to-normal vision.

Experimental Design, Materials, and Procedure. The acquisition phase was identical to that in Experiment 1.

In the test phase, participants were instructed to respond to colored circles (orange or blue) with either a right or a left buttonpress; the color to key mapping was counterbalanced across participants. We again used an intertrial interval of $1,000,1,500$, or $2,000 \mathrm{msec}$ and then presented a white fixation cross on a black background for $100 \mathrm{msec}$, which was followed by a blank screen for $100 \mathrm{msec}$, after which one of the colored circles was presented for $200 \mathrm{msec}$. Either $100 \mathrm{msec}$ before, simultaneously with, or $100 \mathrm{msec}$ after onset of the circle, one of the three effect tones was presented. RTs above $1,500 \mathrm{msec}$ were discarded (Figure 3).

The block structure was identical to that used in Experiment 1.

The data analysis focused mainly on the test phase. We calculated a $3 \times 3$ repeated measures ANOVA with the factors jitter $(-100$, $0,+100 \mathrm{msec}$ ) and tone (compatible action, incompatible action, nonaction) on the RTs.

\section{Results and Discussion}

In the acquisition phase, participants produced, on average, the same number of right, left, and no buttonpresses (right, 136; left, 134; intentional nonaction, 135), confirming that the participants experienced the actioneffect couplings about equally often.

We had to exclude $0.02 \%$ of the trials because participants responded either too late or wrong.

The repeated measures ANOVA with RT as dependent variable resulted in a significant main effect of tone $[F(1,13)=5.10, p<.05]$, indicating that the presentation of the nonaction as well as the incompatible action tone leads to increased RTs. The main effect of jitter $[F(1,13)=$ $8.89, p<.01]$, being based on faster responses when the tone was presented before as opposed to after the circle, was also significant. The interaction of tone and jitter was not significant $[F(1,13)=0.56, p>.05]$ (see Figure 4). In post hoc paired $t$ tests, we found significant differences between the tones when averaging about the factor jitter. 


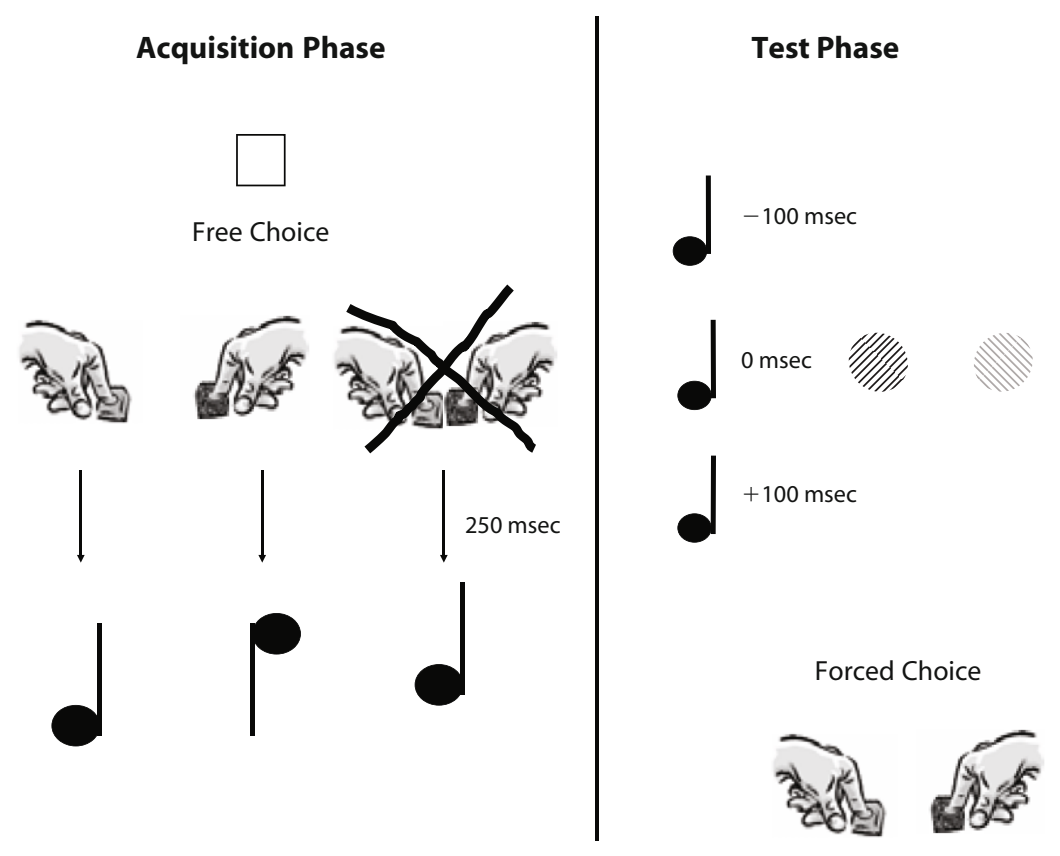

Figure 3. Schematic drawing of the conditions in Experiment 2.

Namely, responding when a compatible tone was presented was significantly faster than when an incompatible action tone was presented $[t(13)=-2.32, p<.05]$. The same holds true when comparing RTs after a compatible tone was presented with RTs after incompatible stop-tone presentation $[t(13)=-2.54, p<.05]$. When we applied a conservative Bonferroni correction, the latter but most important difference-between RTs after compatible tones and RTs after incompatible stop tones-remained significant.

To summarize, presenting the compatible action tone facilitates RTs, whereas the incompatible nonaction as well as the incompatible action tone increases RTs. These results replicate the nonaction and action-effect binding shown in Experiment 1 while at the same time ruling out that the bias reflects a deliberate strategy of the participants rather than an automatic induced response activation.

Although Experiments 1 and 2 demonstrate nonactioneffect binding, they do not reveal what exactly is associated to the action effect in the case of nonaction. It is hard to believe that the nonaction-effect binding consists of nothing being associated with the effect tone. Rather, it is more likely that it is the intention not to act that becomes associ-

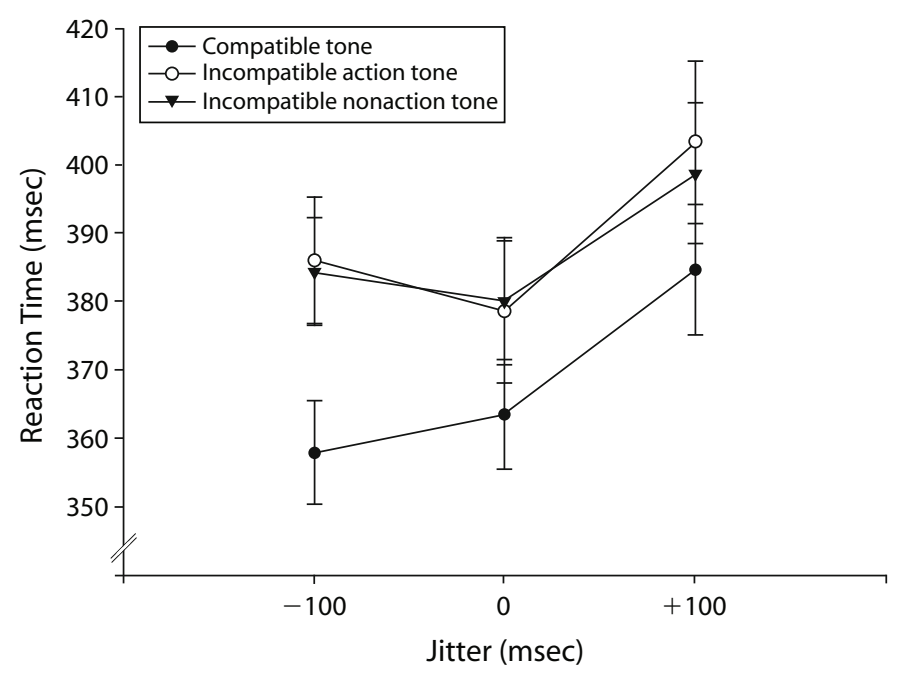

Figure 4. Reaction time (in milliseconds) in response to the presented colored circle in Experiment 2. Error bars represent the standard errors of the means. 


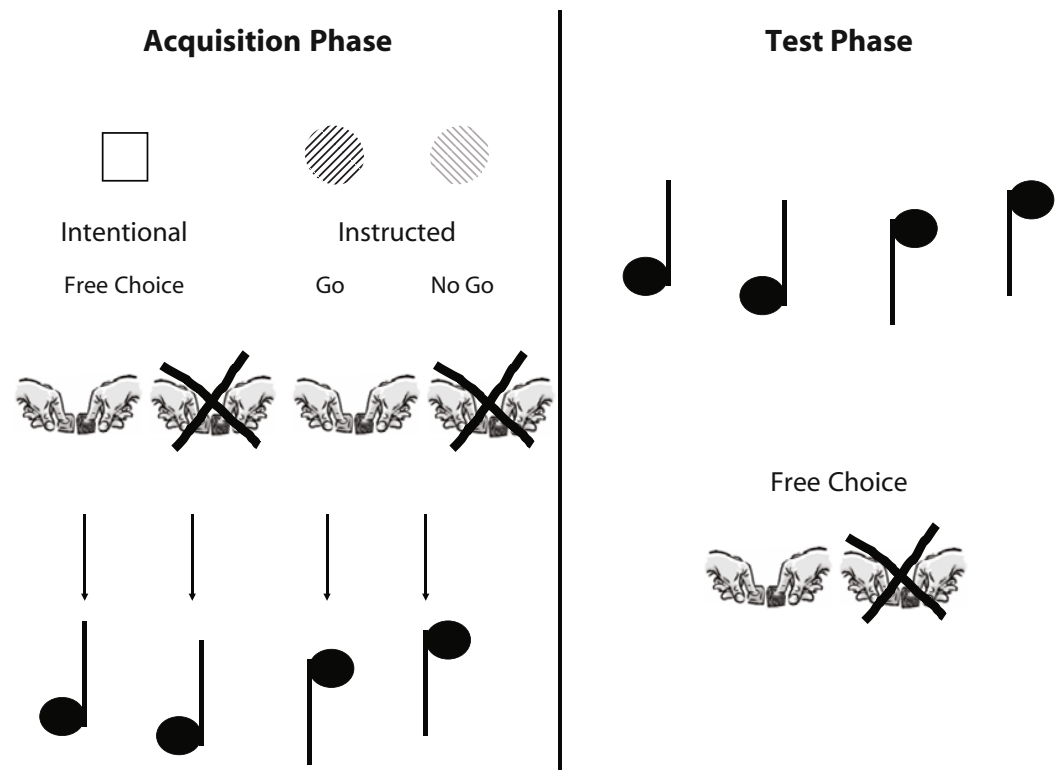

Figure 5. Schematic drawing of the conditions in Experiment 3.

ated with the effect. To further clarify this point, in Experiment 3 we directly compare binding effects of voluntary nonactions, where participants have to form an intention not to act, with those of forced nonactions, where participants are not required to form an intention not to act.

\section{EXPERIMENT 3}

According to associative models (Elsner \& Hommel, 2001), a direct binding between the response and the stimulus is established. It is assumed that motor patterns are bound to the pattern of activation elicited by the effect tone simply due to co-occurrence of both activation patterns. Associative models have problems explaining nonaction-effect binding, as shown in Experiments 1 and 2, because they have to assume a specific motor pattern for nonaction. Moreover, the model predicts that the binding should take place independently of the intentionality of the nonaction.

In contrast to associative models, the anticipative learning model (Ziessler, Nattkemper, \& Frensch, 2004) assumes that the binding between the response and the stimulus is mediated by the anticipated effects on the basis of assumptions of forward and inverse models (Wolpert, Miall, \& Kawato, 1998). Since responses are connected to anticipated effects rather than to the actual effects, actioneffect binding can emerge only if anticipation takes place during action planning. Therefore, the anticipative learning model would predict nonaction-effect binding only for intentional nonactions where the response is planned and the action-effect is actively anticipated. In this sense, there is a principal difference between an externally triggered nonaction and an externally triggered action regarding the involvement of intentions. When an external stimulus indicates that I have to act, I nevertheless have to activate the motor program to do it. In this sense, externally triggered behavior requires a form of anticipation. In contrast, when a stimulus indicates to do nothing, I don't have to form an additional intention to implement this instruction. In this sense, one would expect an asymmetry regarding action-effect binding for externally triggered actions and nonaction.

In order to conclude that the deliberate choice not to act is the characteristic feature causing the binding in the nonaction condition, we compare intentional nonactions with instructed nonactions. The aim of the third experiment therefore was to demonstrate that intentional nonactions do show nonaction-effect binding, whereas instructed nonactions do not.

\section{Method}

Participants. Fifteen individuals ( 8 females and 7 males; ages 19-26 years) took part in this experiment to obtain course credit. All participants were right-handed and had normal or corrected-tonormal vision.

Experimental Design, Materials, and Procedure. Experiment 3 was similar to Experiment 1, with the difference that participants were instructed to decide between pressing two buttons simultaneously (intentional "go") with their right and left index fingers or not pressing both buttons (intentional "no go") in the acquisition phase. As in Experiment 1, the participants were asked to aim at an equal distribution of responding and not responding with each effector, without having a certain rule of responding. We asked the participants to flip an imaginary coin in their minds at the moment when the white square appeared. We also used two colored circles (blue or orange) of a size similar to that of the white square as stimuli. Half of the participants were instructed to press both buttons when a blue circle appeared (instructed "go") and to press no button when an orange circle appeared (instructed "no go") (see Figure 5).

For the other half of the participants, the task mapping for the blue and orange circle was reversed. In $50 \%$ of the trials, white squares, requiring an intentional choice, were presented; in the remaining trials, orange or blue circles were presented with an equal probability. This 


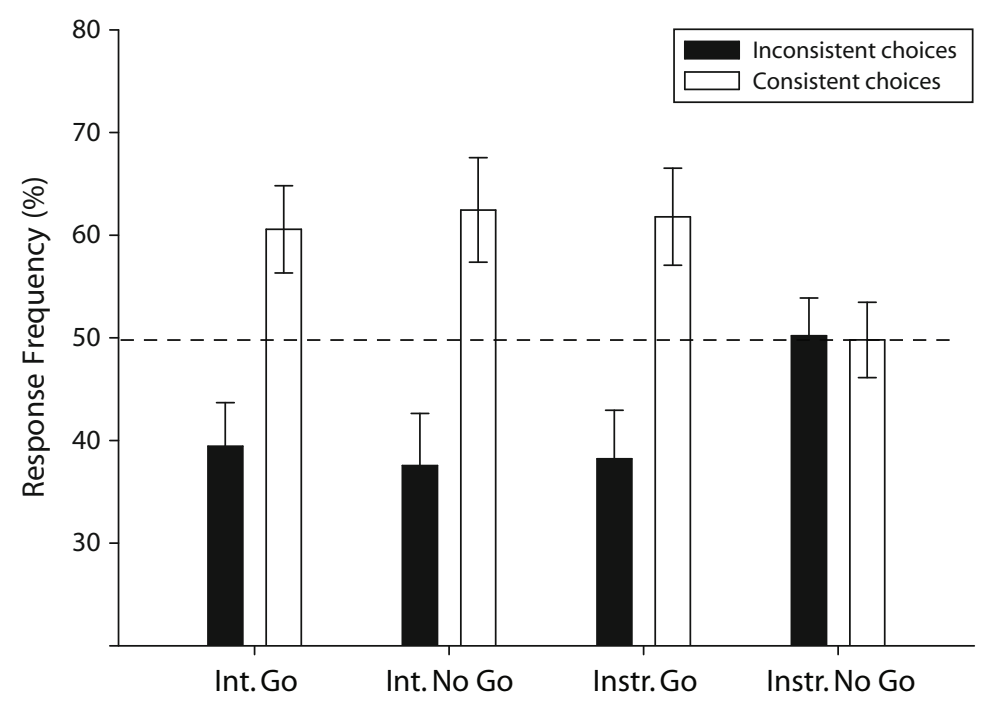

Figure 6. Response frequency in response to the presented tone in Experiment 3. Error bars represent the standard errors of the means.

distribution of trials assures the same amount of actions and nonactions and likewise prohibits any systematic response preparation.

In contrast to Experiment 1, we used four everyday noises (telephone ringing, glass breaking, computer jingle, nose blowing) of 200-msec duration to make the sounds more distinguishable.

In the test phase, the participants heard one of the four effect sounds and were supposed to choose freely between pressing and not pressing both response buttons. If the response occurred during the tone presentation, the feedback "Too early!" was presented on the screen and those trials were excluded from further analysis.

Each block consisted of 48 trials; the experiment contained 15 blocks. Apart from these differences, everything else was identical to Experiment 1.

\section{Results and Discussion}

In the acquisition phase, participants produced, on average, the same number of valid intentional action and nonaction trials (intentional action, 104; intentional nonaction, 105; on average, 7 intentional action trials had to be excluded because participants responded too late) and the same number of valid instructed action and nonaction trials (instructed action, 108; instructed nonaction, 104 ; on average, 4 instructed nonaction trials had to be excluded because a response was given) after the intentional nonaction tone had already been presented.

We excluded $0.09 \%$ of the test phase trials because the participants responded too early. KS tests revealed no violations of the assumption of normal distribution ( $p>.39$ for intentional "go," intentional "no go," instructed "go," and instructed "no go" frequency of consistent choices). $t$ tests revealed that the frequency of consistent choices deviated significantly from chance: the intentional "go" $[t(14)=2.48, p<.05]$, the intentional "no go" $[t(14)=$ $2.45, p<.05]$, and the instructed "go" tone $[t(14)=2.49$, $p<.05]$ (see Figure 6).

As predicted, the consistent choices were not significantly different from chance for the instructed "no go" tone; $t$ test results were clearly not significant $[t(14)=$ $-0.06, p=.96]$. A repeated measures ANOVA involving only the two different kinds of nonactions reveals a significant interaction effect of the factors consistency and deliberateness $[F(1,14)=6.83, p<.05]$.

To summarize, we find effect binding in both intentional conditions and in the instructed action condition but not in the instructed nonaction condition. This supports the view that the characteristic feature shared by voluntary as well as instructed actions and voluntary nonaction is the intention formation, which is present in all three conditions exhibiting the binding effect.

\section{GENERAL DISCUSSION}

In the presented experiments, we demonstrated actioneffect binding for voluntary not-acting. In the first experiment, associations between right, left, and no buttonpresses and three tones were learned and the binding was demonstrated in frequencies of choice. The second experiment replicated the effect binding of voluntary nonactions and actions by means of RTs. The third experiment revealed differences in binding between voluntary nonactions and instructed nonactions, pointing to the fact that the characteristic feature of the binding is the voluntariness of the choice, not the nonaction per se. These results support our claim that deliberate nonactions are based on representations similar to intentional actions and can likewise develop an association with the subsequent effect tone.

\section{Nonaction-Effect Binding?}

Action-effect binding has been described as the result of automatic processes in which associations between "motor patterns" in the "motor system" (Elsner \& Hommel, 2001, p. 230; based on Lotze, 1852) and the representations of the perceivable effects in the "cognitive system" are established. However, a study by Ziessler and Nattkemper (2002) demonstrated that an overt execution 
of a response in the acquisition phase is not required to produce action-effect binding in a go/no-go context. The authors show that action planning processes are sufficient to produce binding between responses and their sensory consequences. Our data further extend these findings by showing action-effect binding for intentional nonaction (Experiments 1 and 2). Experiment 3 suggests that it is not the absence of an overt response but the intention not to act that is bound to the tone. But what is the content of such intentions? According to ideomotor theory, the most parsimonious interpretation of the content of such intentions would be that they represent the prediction of the desired end state of a behavior or nonbehavior. Regarding proximal predictions of a movement, such predicted end states necessarily have to differ. The intention to move would involve the prediction of a change in the movement-related feedback (i.e., proprioceptive information). In contrast, intending not to move would predict no change in the proximal consequences. However, regarding distal consequences (i.e., effect tones), the intentions to move or not to move do not necessarily differ. Intending not to move can lead to a prediction of changes in the environment, just like intending to move. This is why we assume that the binding occurs between the intention to respond or not to respond and the effect tone. This is in line with the anticipative learning model (Ziessler et al., 2004), suggesting that responses are not connected to the actual effects but rather to the anticipated effects that constitute the motor intention. Our third experiment clearly demonstrates that an active mental process - namely, an intention, or, put differently, the anticipated effect-is necessary to produce nonaction-effect binding, because the omission of an action alone (instructed "no go") is not sufficient to produce nonaction-effect binding.

The binding effect we find for instructed actions seems to be at odds with a recent study by Herwig, Prinz, and Waszak (2007). The authors suggested that instructed actions are accompanied by $\mathrm{S}-\mathrm{R}$ learning and therefore do not produce action-effect binding, whereas intentional actions result in R-S-like action-effect binding. The following differences between the studies might explain the differences in results: Herwig et al. employed a test phase in which they compared RTs in compatible versus incompatible responses between participants, whereas we use a free-choice task and compare within participants. Unlike in a choice RT task, participants experience the lack of a specific goal to drive their choice in our task; therefore, the task used here might be especially sensitive for measuring even slight response tendencies.

\section{Representation of Nonaction}

Wittgenstein (1953) raised the ingenious question, "When I raise my arm, what is left after subtracting the fact that my arm goes up?"' (p. 621). Jeannerod (1999) answered this query by stating that an internal model of the planned action is left. In the present context, the equivalent question would be, "When I don't raise my arm, what is left if I intend not to raise my arm?" One possible answer would be that the same internal model of the possible arm movement without the overt action component is left. However, our results render this interpretation rather unlikely. The representation of a nonaction cannot be believed to be the same as the representation of an action without the accompanied action, because then we would not expect that different tones are associated with a specific action and the corresponding omission of that action. There are two interpretations that would be in accordance with the present results. The first interpretation suggests that the representation of nonaction comprises the top-down inhibition of the motor representation of the not-executed act. This would suggest that the inhibition component would be specific for nonactions. However, the alternative assumption is similar to the concept of the representation of negations in memory (Gilbert, 1991). This account would suggest that representations of nonactions contain the abstract representation of the not-executed action, "tagged" as false.

The present data do not support the inhibition hypothesis. When taking into account transitions between trials in the acquisition phase, RTs in instructed action trials are significantly faster following intentional nonactions $(270 \mathrm{msec})$ as compared with intentional actions $(310 \mathrm{msec})[t(14)=-5.34, p<.001]$ and even compared with repetition trials in which an instructed action is followed by the same instructed action $(282 \mathrm{msec})[t(14)=$ $-2.30, p<.05]$. The inhibition account would predict the opposite pattern: The suppression of an action should lead to slower RTs in the next trial, because the inhibition needs to be overcome.

We cannot explicitly decide between these two alternative forms of representation with the present data, but the inhibition hypothesis leads to easily testable predictions that can be addressed in further research by use of neuroimaging methods.

To conclude, the fact that research primarily focuses on overt action seems to be a relic of the behavioristic conception that needs to be overcome. Surely, having no overt response measure is a clear disadvantage, but in the long run, exploring intentional nonaction might offer a new prospect for understanding action and might eventually give us an understanding of the wei wu wei (acting by not acting) paradox of Taoism, which considers not acting to be a mode of action.

\section{AUTHOR NOTE}

The work was supported by a dissertation grant from the Studienstiftung des Deutschen Volkes, awarded to the first author. Correspondence concerning this article should be addressed to S. Kühn, Faculty of Psychology and Educational Sciences, Department of Experimental Psychology, Ghent University, Henri Dunantlaan 2, 9000 Ghent, Belgium (e-mail: skuehn@cbs.mpg.de).

\section{REFERENCES}

Elsner, B., \& Hommel, B. (2001). Effect anticipation and action control. Journal of Experimental Psychology: Human Perception \& Performance, 27, 229-240.

Gilbert, D. T. (1991). How mental systems believe. American Psychologist, 46, 107-119.

Greenwald, A. G. (1970). A choice reaction time test of ideomotor theory. Journal of Experimental Psychology, 86, 20-25.

Herwig, A., Prinz, W., \& Waszak, F. (2007). Two modes of sensori- 
motor integration in intention-based and stimulus-based actions. Quarterly Journal of Experimental Psychology, 60, 1540-1554.

JEANNEROD, M. (1999). To act or not to act: Perspectives on the representation of actions. Quarterly Journal of Experimental Psychology, 52A, 1-29.

Lotze, R. H. (1852). Medicinische Psychologie oder Physiologie der Seele [Medical psychology or physiology of the soul]. Leipzig: Weidmann'sche Buchhandlung.

Loy, D. (1985). Wei-wu-wei: Nondual action. Philosophy East \& West, 35, 73-86.

PrinZ, W. (1997). Perception and action planning. European Journal of Cognitive Psychology, 9, 129-154.

Wittgenstein, L. (1953). Philosophical investigations, I (G. E. M. Anscombe, Trans.). Oxford: Blackwell.

Wolpert, D. M., Miall, R. C., \& Kawato, M. (1998). Internal models in the cerebellum. Trends in Cognitive Sciences, 2, 338-347.

Ziessler, M., \& NATTKEMPER, D. (2002). Effect anticipation in action planning. In W. Prinz \& B. Hommel (Eds.), Common mechanisms in perception and action: Attention and performance XIX (pp. 645-672). Oxford: Oxford University Press.
Ziessler, M., NattKemPer, D., \& Frensch, P. A. (2004). The role of anticipation and intention in the learning of effects of self-performed action. Psychological Research, 68, 163-175.

\section{NOTE}

1. We use the term intentional nonaction to describe a deliberately chosen voluntary refraining from an action, which we want to distinguish from inaction or inactivity, where no distinct intention not to act exists. In the context of our experiments, intentional nonaction is a predefined response option with the special feature that it is not related to an overt movement. Intentional nonactions are different from nonactions in go/ no-go or stop tasks because those work with prepotent response tendencies and external stimuli determining at what point in time inhibition is demanded.

(Manuscript received July 10, 2008; revision accepted for publication December 16, 2008.) 